\title{
Explicit moduli spaces for congruences of elliptic curves
}

\author{
Tom Fisher ${ }^{1}$
}

Received: 28 September 2018 / Accepted: 8 August 2019 / Published online: 14 September 2019 (c) The Author(s) 2019

\begin{abstract}
We determine explicit birational models over $\mathbb{Q}$ for the modular surfaces parametrising pairs of $N$-congruent elliptic curves in all cases where this surface is an elliptic surface. In each case we also determine the rank of the Mordell-Weil lattice and the geometric Picard number.
\end{abstract}

Keywords Elliptic curves · Galois representations · Elliptic surfaces

Mathematics Subject Classification $11 \mathrm{G} 05 \cdot 11 \mathrm{~F} 80 \cdot 14 \mathrm{~J} 27$

\section{Introduction}

Let $N \geq 2$ be an integer. A pair of elliptic curves are said to be $N$-congruent, if their $N$-torsion subgroups are isomorphic as Galois modules. Such an isomorphism raises the Weil pairing to the power $\varepsilon$ for some $\varepsilon \in(\mathbb{Z} / N \mathbb{Z})^{\times}$. In this situation we say that the $N$-congruence has power $\varepsilon$. Since multiplication-by- $m$ on one of the elliptic curves (for $m$ an integer coprime to $N$ ) changes $\varepsilon$ to $m^{2} \varepsilon$, we are only ever interested in the class of $\varepsilon \in(\mathbb{Z} / N \mathbb{Z})^{\times} \bmod$ squares.

Congruences of elliptic curves are interesting for several reasons. They can be used in the modular approach to solving Diophantine equations, in studying morphisms from a genus 2 curve to an elliptic curve, and in visualising elements of the Tate-Shafarevich group. See for example $[9,15,25]$.

Let $Z(N, \varepsilon)$ be the surface that parametrises pairs of $N$-congruent elliptic curves with power $\varepsilon$, up to simultaneous quadratic twist. This is a surface defined over $\mathbb{Q}$. We only consider $Z(N, \varepsilon)$ up to birational equivalence. Kani and Schanz [19, Theorem 4] classified the geometry of these surfaces, explicitly determining the pairs $(N, \varepsilon)$ for which $Z(N, \varepsilon)$ is birational over $\mathbb{C}$ to either (i) a rational surface, (ii) an elliptic $K 3$-surface, (iii) an elliptic surface with Kodaira dimension one (also known as a properly elliptic surface), or (iv) a surface of general type. In case (i) it is known that the surface is rational over $\mathbb{Q}$. We show in cases (ii) and (iii) that the surface is birational over $\mathbb{Q}$ to an elliptic surface, determining in each case a Weierstrass equation for the generic fibre as an elliptic curve over $\mathbb{Q}(T)$. One

Tom Fisher

T.A.Fisher@dpmms.cam.ac.uk

1 Centre for Mathematical Sciences, University of Cambridge, DPMMS, Wilberforce Road,

Cambridge CB3 0WB, UK 
application of these explicit equations is that we are then able to use the methods of van Luijk and Kloosterman to compute the geometric Picard number of each surface.

The problem of computing $Z(N, \varepsilon)$ is closely related to that of computing the modular curves $X_{E}(N, \varepsilon)$ parametrising the elliptic curves $N$-congruent (with power $\varepsilon$ ) to a given elliptic curve $E$. Equations for $X_{E}(N, \varepsilon)$, and the family of curves it parametrises, have been determined as follows. In each case the equations have coefficients in $\mathbb{Q}(a, b)$ where $a$ and $b$ are the coefficients of a Weierstrass equation for $E$. The cases $N \leq 5$ were treated by Rubin and Silverberg [26,28,30] for $\varepsilon=1$, and by Fisher [11,12] for $\varepsilon \neq 1$. The case $N=7$ was treated by Halberstadt and Kraus [17] for $\varepsilon=1$, and by Poonen, Schaefer and Stoll [25] for $\varepsilon \neq 1$. The case $N=8$ was treated by Chen [8], and the cases $N=9$ and $N=11$ by Fisher $[13,14]$.

If $N$ is not a prime power, then in principle we obtain equations for $X_{E}(N, \varepsilon)$ as a fibre product of modular curves of smaller level. Equations that are substantially better than this have been obtained in the case $(N, \varepsilon)=(6,1)$ by Rubin and Silverberg [27], and independently Papadopoulos [24], and in the cases $(N, \varepsilon)=(12,1)$ and $(12,7)$ by Chen [7, Chapter 7]. Chen also gives equations for $X_{E}(N, \varepsilon)$ in the cases $(N, \varepsilon)=(6,5)$ and $(10,1)$.

The equations for $X_{E}(N, \varepsilon)$ do immediately give us equations for $Z(N, \varepsilon)$, but unfortunately this does not always make it easy to find the elliptic fibrations. The main purpose of this note is to record the transformations that work in each case.

According to [19, Theorem 4] the surface $Z(N, \varepsilon)$ is rational over $\mathbb{C}$ for all $N \leq 5$, and in the cases $N=6,7,8$ with $\varepsilon=1$. In each of these cases $Z(N, \varepsilon)$ is rational over $\mathbb{Q}$, as follows (see [7, Chapter 8]) from the results cited above.

In our terminology, it is part of the definition of an elliptic surface that it has a section. As we describe below, some of the cases in the next two theorems were already treated in $[7,8,14,21]$.

Theorem 1.1 The surfaces $Z(N, \varepsilon)$ that are birational over $\mathbb{C}$ to an elliptic $K 3$-surface, are in fact birational over $\mathbb{Q}$ to an elliptic surface. The generic fibres are the elliptic curves over $\mathbb{Q}(T)$ with the following Weierstrass equations.

$$
\begin{array}{ll}
Z(6,5): & y^{2}+3 T(T-2) x y+2(T-1)(T+2)^{2}\left(T^{3}-2\right) y=x^{3}-6(T-1)\left(T^{3}-2\right) x^{2}, \\
Z(7,3): & y^{2}=x^{3}+\left(4 T^{4}+4 T^{3}-51 T^{2}-2 T-50\right) x^{2}+(6 T+25)\left(52 T^{2}-4 T+25\right) x, \\
Z(8,3): & y^{2}=x^{3}-\left(3 T^{2}-7\right) x^{2}-4 T^{2}\left(4 T^{4}-15\right) x+4 T^{2}\left(53 T^{4}+81 T^{2}+162\right), \\
Z(8,5): & y^{2}=x^{3}-2\left(T^{2}+19\right) x^{2}-\left(4 T^{2}-49\right)\left(T^{4}-6 T^{2}+25\right) x, \\
Z(9,1): & y^{2}+\left(6 T^{2}+3 T+2\right) x y+T^{2}(T+1)\left(4 T^{3}+9 T+9\right) y \\
& =x^{3}-\left(16 T^{4}+12 T^{3}+9 T^{2}+6 T+1\right) x^{2}, \\
Z(12,1): & y^{2}+2\left(5 T^{2}+9\right) x y+96\left(T^{2}+3\right)\left(T^{2}+1\right)^{2} y=x^{3}+\left(T^{2}+3\right)\left(11 T^{2}+1\right) x^{2} .
\end{array}
$$

Theorem 1.2 The surfaces $Z(N, \varepsilon)$ that are birational over $\mathbb{C}$ to a properly elliptic surface, are in fact birational over $\mathbb{Q}$ to an elliptic surface. The generic fibres are the elliptic curves over $\mathbb{Q}(T)$ with the following Weierstrass equations.

$$
\begin{array}{ll}
Z(8,7): & y^{2}=x^{3}+2\left(4 T^{6}-15 T^{4}+14 T^{2}-1\right) x^{2}+\left(T^{2}-1\right)^{4}\left(16 T^{4}-24 T^{2}+1\right) x, \\
Z(9,2): & y^{2}+3\left(4 T^{3}+T^{2}-2\right) x y+(T-1)^{3}\left(T^{3}-1\right)\left(4 T^{3}-3 T-7\right) y \\
& =x^{3}-3(T+1)\left(T^{3}-1\right)\left(9 T^{2}+2 T+1\right) x^{2}, \\
Z(10,1): & y^{2}-(3 T-2)\left(6 T^{2}-5 T-2\right) x y
\end{array}
$$




$$
\begin{array}{ll} 
& -4 T^{2}(T-1)^{2}\left(4 T^{2}-2 T-1\right)\left(27 T^{3}-54 T^{2}+16 T+12\right) y \\
& =x^{3}+T^{2}(T-1)\left(27 T^{3}-54 T^{2}+16 T+12\right) x^{2}, \\
Z(10,3): \quad & y^{2}+\left(T^{3}-8 T^{2}-9 T-8\right) x y+2 T^{2}\left(T^{3}-T^{2}-3 T-3\right)\left(7 T^{2}+2 T+3\right) y \\
= & x^{3}+2(3 T+2)\left(T^{3}-T^{2}-3 T-3\right) x^{2}, \\
Z(11,1): \quad & y^{2}+\left(T^{3}+T\right) x y=x^{3}-\left(4 T^{5}-17 T^{4}+30 T^{3}-18 T^{2}+4\right) x^{2} \\
+ & T^{2}(2 T-1)\left(3 T^{2}-7 T+5\right)^{2} x .
\end{array}
$$

Although we have not made it formally part of the statements of Theorems 1.1 and 1.2, our methods do also give the moduli interpretations of these surfaces. In other words, given a point on one of these surfaces (away from a certain finite set of curves) we can determine the corresponding pair of $N$-congruent elliptic curves. The fact that $N$-congruent elliptic curves over $\mathbb{Q}$ have traces of Frobenius (at all primes of good reduction) that are congruent mod $N$, then provides some very useful check on our calculations. See for example [7, Chapter 9] or the calculations at the end of the Magma file accompanying this article.

The second part of the following corollary was conjectured by Kani and Schanz [19, Conjecture 5], and its proof (for $\varepsilon=1$ ) was completed by Zexiang Chen in his $\mathrm{PhD}$ thesis [7]. For $N$ sufficiently large it is expected (with variants of this conjecture variously attributed to Frey, Mazur, Kani and Darmon) that the conclusions of the corollary are false.

Corollary 1.3 Let $N \leq 12$ and $\varepsilon \in(\mathbb{Z} / N \mathbb{Z})^{\times}$with $\varepsilon=1$ if $N=11$ or 12 .

(i) There are infinitely many pairs of non-isogenous elliptic curves over $\mathbb{Q}(T)$ that are $N$-congruent with power $\varepsilon$.

(ii) There are infinitely many pairs of non-isogenous elliptic curves over $\mathbb{Q}$ that are $N$ congruent with power $\varepsilon$.

Moreover the $j$-invariants $j_{1}$ and $j_{2}$ of the elliptic curves in (i) (resp. (ii)) correspond to infinitely many curves (resp. points) in the $\left(j_{1}, j_{2}\right)$-plane.

Proof In Table 3 we list at least one $\mathbb{Q}$-rational section of infinite order for each of the elliptic surfaces in Theorems 1.1 and 1.2. This proves the first part. The second part follows by specialising $T$. See the proof of [14, Theorem 1.5] for further details. The final sentence of the statement is included to guard against various "cheat" proofs, where new examples are generated from old by taking quadratic twists, or making substitutions for $T$.

Remark 1.4 If elliptic curves $E_{1}$ and $E_{2}$ are $N$-congruent with power $\varepsilon=-1$, then the quotient of $E_{1} \times E_{2}$ by the graph of the $N$-congruence is a principally polarised abelian surface. The surface $Z(N,-1)$ may then be interpreted as a Hilbert modular surface, parametrising degree $N$ morphisms from a genus 2 curve to an elliptic curve. At the outset of our work, this moduli interpretation had not been made explicit for any $N>5$. Remarkably however, this approach has been used by A. Kumar [21] to independently obtain results equivalent to the first two parts of Theorem 1.1 and the first three parts of Theorem 1.2. As far as we are aware, his methods do not generalise to $\varepsilon \neq-1$.

In Tables 1 and 2 we record some further data concerning the elliptic surfaces in Theorems 1.1 and 1.2. It is well known that K3-surfaces may admit more than one elliptic fibration. For some interesting arithmetic applications see [10,31,35]. In view of this, the data in Table 1 comes with the caveat that it relates to the elliptic fibration we happened to find in Theorem 1.1. Since a properly elliptic surface has a unique elliptic fibration, there is no such caveat 
Table 1 The elliptic K3-surfaces in Theorem 1.1

\begin{tabular}{|c|c|c|c|c|c|}
\hline$(N, \varepsilon)$ & Singular fibres & |tors & $\operatorname{rank} / \mathbb{Q}$ & $\operatorname{rank} / \overline{\mathbb{Q}}$ & $\rho$ \\
\hline$(6,5)$ & $\left(\mathrm{I}_{2}, \mathrm{I}_{2}\right), \mathrm{I}_{3},\left(\mathrm{I}_{3}, \mathrm{I}_{3}, \mathrm{I}_{3}\right), \mathrm{I}_{4}, \mathrm{I}_{4}$ & 1 & 2 & 2 & 20 \\
\hline$(7,3)$ & $\mathrm{I}_{1}, \mathrm{I}_{2},\left(\mathrm{I}_{2}, \mathrm{I}_{2}\right),\left(\mathrm{I}_{2}, \mathrm{I}_{2}\right), \mathrm{I}_{3}, \mathrm{I}_{10}$ & 2 & 2 & 2 & 20 \\
\hline$(8,3)$ & $\left(\mathrm{I}_{1}, \mathrm{I}_{1}\right), \mathrm{I}_{2},\left(\mathrm{I}_{2}, \mathrm{I}_{2}\right),\left(\mathrm{I}_{2}, \mathrm{I}_{2}\right),\left(\mathrm{I}_{3}, \mathrm{I}_{3}\right), \mathrm{I}_{0}^{*}$ & 1 & 4 & 5 & 20 \\
\hline$(8,5)$ & $\mathrm{I}_{2}, \mathrm{I}_{2},\left(\mathrm{I}_{2}, \mathrm{I}_{2}\right),\left(\mathrm{I}_{2}, \mathrm{I}_{2}\right),\left(\mathrm{I}_{3}, \mathrm{I}_{3}\right), \mathrm{I}_{0}^{*}$ & 2 & 2 & 4 & 20 \\
\hline$(9,1)$ & $\left(\mathrm{I}_{1}, \mathrm{I}_{1}, \mathrm{I}_{1}\right), \mathrm{I}_{2},\left(\mathrm{I}_{2}, \mathrm{I}_{2}, \mathrm{I}_{2}\right), \mathrm{I}_{3}, \mathrm{I}_{4}, \mathrm{I}_{0}^{*}$ & 1 & 3 & 4 & 19 \\
\hline$(12,1)$ & $\left(\mathrm{I}_{1}, \mathrm{I}_{1}, \mathrm{I}_{1}, \mathrm{I}_{1}, \mathrm{I}_{1}, \mathrm{I}_{1}, \mathrm{I}_{1}, \mathrm{I}_{1}\right),\left(\mathrm{I}_{4}, \mathrm{I}_{4}\right),\left(\mathrm{I}_{4}, \mathrm{I}_{4}\right)$ & 1 & 3 & 5 & 19 \\
\hline
\end{tabular}

Table 2 The properly elliptic surfaces in Theorem 1.2

\begin{tabular}{llllll}
\hline$(N, \varepsilon)$ & Singular fibres & $\mid$ tors $\mid$ & rank $/ \mathbb{Q}$ & rank $/ \overline{\mathbb{Q}}$ & $\rho$ \\
\hline$(8,7)$ & $\mathrm{I}_{2},\left(\mathrm{I}_{2}, \mathrm{I}_{2}\right),\left(\mathrm{I}_{2}, \mathrm{I}_{2}\right),\left(\mathrm{I}_{3}, \mathrm{I}_{3}\right), \mathrm{I}_{4}, \mathrm{I}_{8}, \mathrm{I}_{8}$ & 2 & 1 & 2 & 30 \\
$(9,2)$ & $\left(\mathrm{I}_{2}, \mathrm{I}_{2}, \mathrm{I}_{2}\right),\left(\mathrm{I}_{3}, \mathrm{I}_{3}\right),\left(\mathrm{I}_{3}, \mathrm{I}_{3}, \mathrm{I}_{3}\right), \mathrm{I}_{9}, \mathrm{I}_{0}^{*}$ & 1 & 2 & 2 & 29 \\
$(10,1)$ & $\left(\mathrm{I}_{2}, \mathrm{I}_{2}\right),\left(\mathrm{I}_{2}, \mathrm{I}_{2}\right),\left(\mathrm{I}_{3}, \mathrm{I}_{3}, \mathrm{I}_{3}\right), \mathrm{I}_{5}, \mathrm{I}_{10}, \mathrm{IV}$ & 1 & 1 & 1 & 28 \\
$(10,3)$ & $\left(\mathrm{I}_{1}, \mathrm{I}_{1}, \mathrm{I}_{1}\right), \mathrm{I}_{2},\left(\mathrm{I}_{2}, \mathrm{I}_{2}\right),\left(\mathrm{I}_{2}, \mathrm{I}_{2}\right),\left(\mathrm{I}_{3}, \mathrm{I}_{3}, \mathrm{I}_{3}\right), \mathrm{I}_{4}, \mathrm{I}_{4}, \mathrm{I}_{6}$ & 1 & 3 & 4 & 28 \\
$(11,1)$ & $\left(\mathrm{I}_{1}, \mathrm{I}_{1}, \mathrm{I}_{1}\right), \mathrm{I}_{2},\left(\mathrm{I}_{2}, \mathrm{I}_{2}, \mathrm{I}_{2}\right), \mathrm{I}_{3}, \mathrm{I}_{4},\left(\mathrm{I}_{4}, \mathrm{I}_{4}\right), \mathrm{I}_{10}$ & 2 & 2 & 2 & 28 \\
\hline
\end{tabular}

Table 3 Mordell-Weil generators over $\mathbb{Q}(T)$

\begin{tabular}{ll}
\hline$(N, \varepsilon)$ & $x$-coordinates of independent sections of infinite order \\
\hline$(6,5)$ & $0,2 T^{4}-4 T$, \\
$(7,3)$ & $4 T^{2}+20 T+25,6 T+25$, \\
$(8,3)$ & $-7,-T^{2}+9,-4 T^{2}-6 T,\left(4 T^{5}-2 T^{4}+10 T^{3}+6 T^{2}+18 T\right) /(T-1)^{2}$, \\
$(8,5)$ & $-4 T^{2}+49,2 T^{3}+19 T^{2}+60 T+63$, \\
$(9,1)$ & $0,4 T^{4}+2 T^{3}-2 T^{2}, 4 T^{4}+4 T^{3}+9 T^{2}+18 T+9$, \\
$(12,1)$ & $0,-12 T^{4}-24 T^{2}-12,4 T^{6}+12 T^{4}-4 T^{2}-12$, \\
$(8,7)$ & $4 T^{6}+4 T^{5}-9 T^{4}-10 T^{3}+4 T^{2}+6 T+1$, \\
$(9,2)$ & $0,2 T^{5}-8 T^{3}+4 T^{2}+6 T-4$, \\
$(10,1)$ & 0, \\
$(10,3)$ & $0,2 T^{5}-4 T^{4}-4 T^{3}+6 T, 4 T^{5}-2 T^{4}-14 T^{3}-18 T^{2}-6 T$, \\
$(11,1)$ & $T^{4}+4 T^{2}+4,3 T^{2}-7 T+5$. \\
\hline
\end{tabular}

for Table 2. We list in each case the Kodaira symbols of the singular fibres (with bracketing to indicate fibres that are Galois conjugates), the order of the torsion subgroup over $\mathbb{Q}(T)$, the ranks of the group of sections over $\mathbb{Q}(T)$ and $\overline{\mathbb{Q}}(T)$, and finally the geometric Picard number $\rho$. The lower bounds on the ranks are immediate from the independent sections of infinite order listed in Tables 3 and 4. The upper bounds on the ranks, and the geometric Picard numbers are justified in Sect. 4.

We organise the proofs of Theorems 1.1 and 1.2 as follows. The cases $N=8$ and $N=9$ were already treated in $[8,14]$, by starting from equations for $X_{E}(N, \varepsilon)$. In Sect. 2 we use a similar approach to treat the cases $(N, \varepsilon)=(7,3),(11,1)$ and $(12,1)$. Then in Sect. 3 we treat the cases $(N, \varepsilon)=(6,5),(10,1)$ and $(10,3)$ by exhibiting $Z(N, \varepsilon)$ as a degree 3 cover 
Table 4 Additional Mordell-Weil generators over $\overline{\mathbb{Q}}(T)$

\begin{tabular}{lll}
\hline$(N, \varepsilon)$ & $d$ & Section of infinite order defined over $\mathbb{Q}(\sqrt{d})$ \\
\hline$(8,3)$ & -2 & $\left(-2 T^{4}-5 T^{2}-9,\left(2 T^{6}+5 T^{4}+20 T^{2}+9\right) \sqrt{-2}\right)$, \\
$(8,5)$ & -3 & $\left(-2 T^{3}+T^{2}+18 T-35,\left(12 T^{3}-6 T^{2}-108 T+210\right) \sqrt{-3}\right)$, \\
$(8,5)$ & -1 & $\left(16 T^{2}-196,\left(8 T^{4}-346 T^{2}+3038\right) \sqrt{-1}\right)$, \\
$(9,1)$ & -3 & $\left(-(19 / 3) T^{4}-15 T^{3}-9 T^{2}, \ldots\right)$, \\
$(12,1)$ & -3 & $\left(-12 T^{4}-40 T^{2}-12,\left(8 T^{5}+48 T^{3}+72 T\right) \sqrt{-3}+12 T^{6}+68 T^{4}+84 T^{2}-36\right)$, \\
$(12,1)$ & -1 & $\left(-16 T^{4}-64 T^{2}-48,\left(16 T^{6}+112 T^{4}+240 T^{2}+144\right) \sqrt{-1}+32 T^{6}+224 T^{4}+\right.$ \\
& & $\left.480 T^{2}+288\right)$, \\
$(8,7)$ & -3 & $\left(-4 T^{6}-20 T^{5}-39 T^{4}-36 T^{3}-14 T^{2}+1,\left(24 T^{8}+120 T^{7}+234 T^{6}+216 T^{5}+\right.\right.$ \\
& & $\left.\left.84 T^{4}-6 T^{2}\right) \sqrt{-3}\right)$, \\
$(10,3)$ & -3 & $\left(-7 T^{6}-23 T^{5}-30 T^{4}-15 T^{3}-9 T^{2}, \ldots\right)$. \\
\hline
\end{tabular}

of a K3-surface. See the opening paragraphs of these sections for some more explanation as to how our work relates to that in Chen's thesis [7].

The calculations described in this paper were carried out using Magma [3]. Accompanying Magma files are available from the author's website. We assume that the reader is familiar with the standard techniques for putting an elliptic curve in Weierstrass form, as described in $[4, \S 8]$, or as implemented in Magma.

\section{Proofs via equations for $X_{E}(N, \varepsilon)$}

We prove Theorems 1.1 and 1.2 in the cases $(N, \varepsilon)=(7,3),(11,1)$ and $(12,1)$. The case $(N, \varepsilon)=(7,3)$ was treated in [7, Section 8.2], but as this has not been published before, we include the details for completeness.

Equations for $Z(N, \varepsilon)$ as a surface fibred over the $j$-line may be obtained from the equations for $X_{E}(N, \varepsilon)$ by setting $a=b=-27 j /(4(j-1728))$. However, instead of simply setting $a=b$, it is better to quotient out by the $\mathbb{G}_{m}$-action described below. We obtain the same surface this way since $a$ and $b$ scale under the $\mathbb{G}_{m}$-action by consecutive powers of $\lambda$.

\section{Case $(N, \varepsilon)=(7,3)$}

Let $E$ be the elliptic curve $y^{2}=x^{3}+a x+b$. The following equation for $X_{E}(7,3)$, as a quartic curve in $\mathbb{P}^{2}$, was computed by Poonen, Schaefer and Stoll [25, Section 7.2], building on work of Halberstadt and Kraus [17].

$$
\begin{aligned}
F(a, b ; x, y, z)= & -a^{2} x^{4}+2 a b x^{3} y-12 b x^{3} z-6\left(a^{3}+6 b^{2}\right) x^{2} y^{2}+6 a x^{2} z^{2} \\
& +2 a^{2} b x y^{3}-12 a b x y^{2} z+18 b x y z^{2}+\left(3 a^{4}+19 a b^{2}\right) y^{4} \\
& -2\left(4 a^{3}+21 b^{2}\right) y^{3} z+6 a^{2} y^{2} z^{2}-8 a y z^{3}+3 z^{4} .
\end{aligned}
$$

Replacing $E$ by a quadratic twist does not change the isomorphism class of $X_{E}(7,3)$. This is borne out by the identity

$$
F\left(\lambda^{2} a, \lambda^{3} b ; \lambda x, y, \lambda^{2} z\right)=\lambda^{8} F(a, b ; x, y, z) .
$$


The surface $Z(7,3)$ is the quotient of $\{F=0\} \subset \mathbb{A}^{2} \times \mathbb{P}^{2}$ by this $\mathbb{G}_{m}$-action. We have $F\left(y, x_{1} y ; x_{2}, T, y\right)=y^{2}\left(c y^{2}+h y-f\right)$ where

$$
\begin{aligned}
& c=\left(T^{2}+1\right)\left(3 T^{2}-8 T+3\right), \\
& h=T^{3}(19 T-42) x_{1}^{2}+2 T(T-3)^{2} x_{1} x_{2}-6\left(T^{2}-1\right) x_{2}^{2}, \\
& f=36 T^{2} x_{1}^{2} x_{2}^{2}-2(T-6) x_{1} x_{2}^{3}+x_{2}^{4} .
\end{aligned}
$$

Therefore $Z(7,3)$ is birational to the total space (i.e., the corresponding fibred surface) for the genus one curve over $\mathbb{Q}(T)$ with equation $Y^{2}=h^{2}+4 c f$. This is a double cover of $\mathbb{P}^{1}$ with a rational point above $\left(x_{1}: x_{2}\right)=(1: 0)$. Putting this elliptic curve in Weierstrass form, and replacing $T$ by $(6 T-3) /(4 T+4)$, gives the equation in the statement of Theorem 1.1.

\section{Case $(N, \varepsilon)=(11,1)$}

Let $E$ be the elliptic curve $y^{2}=x^{3}+a x+b$. Equations for $X_{E}(11,1)$ as a curve in $\mathbb{P}^{4}$ were computed in [13, Theorem 1.2]. These equations are the $4 \times 4$ minors of the $5 \times 5$ Hessian matrix of the cubic form

$$
\begin{aligned}
F(a, b ; v, w, x, y, z)= & v^{3}+a v^{2} z-2 a v x^{2}+4 a v x y-6 b v x z+a v y^{2}+6 b v y z \\
& +a^{2} v z^{2}-w^{3}+a w^{2} z-4 a w x^{2}-12 b w x z+a^{2} w z^{2}-2 b x^{3}+3 b x^{2} y \\
& +2 a^{2} x^{2} z+6 b x y^{2}+4 a b x z^{2}+b y^{3}-a^{2} y^{2} z+a b y z^{2}+2 b^{2} z^{3} .
\end{aligned}
$$

Replacing $E$ by a quadratic twist does not change the isomorphism class of $X_{E}(11,1)$. This is borne out by the identity

$$
F\left(\lambda^{2} a, \lambda^{3} b ; \lambda^{2} v, \lambda^{2} w, \lambda x, \lambda y, z\right)=\lambda^{6} F(a, b ; v, w, x, y, z) .
$$

We may describe $Z(11,1)$ as the quotient of a 3 -fold in $\mathbb{A}^{2} \times \mathbb{P}^{4}$ by this $\mathbb{G}_{m}$-action.

We start by using the discriminant condition $4 a^{3}+27 b^{2} \neq 0$ to simplify the equations for $X_{E}(11,1)$. The polynomials

$$
\begin{aligned}
& F_{1}=v z+2 w z+x^{2}-x y-y^{2}, \\
& F_{2}=a x z+b z^{2}-v x+v y-2 w x, \\
& F_{3}=a^{2} z^{2}+2 a w z-4 a x^{2}-12 b x z-3 w^{2}, \\
& F_{4}=a^{2} z^{2}+2 a v z-2 a x^{2}+4 a x y+a y^{2}-6 b x z+6 b y z+3 v^{2}, \\
& F_{5}=2 a^{2} y z-a b z^{2}-4 a v x-2 a v y-6 b v z-3 b x^{2}-12 b x y-3 b y^{2},
\end{aligned}
$$

are linear combinations of the derivatives of $F$, where the matrix implicit in taking these linear combinations is invertible if $4 a^{3}+27 b^{2} \neq 0$. Now $X_{E}(11,1)$ is defined by the $4 \times 4$ minors of the $5 \times 5$ Jacobian matrix ( $M$ say) of $F_{1}, \ldots, F_{5}$.

We make the substitutions

$$
\begin{aligned}
a & =\left(4 U+3 x_{3}\right) x_{4}-3 x_{5}^{2}, \\
b & =x_{2}\left(x_{1}+x_{3}\right) x_{4}-\left(4 U+3 x_{3}\right) x_{4} x_{5}+2 x_{5}^{3}, \\
(v, w, x, y, z) & =\left(x_{2} x_{4}+x_{4} x_{5}+x_{5}^{2}, x_{3} x_{4}-x_{5}^{2}, x_{5}, x_{4}, 1\right) .
\end{aligned}
$$

We have $4 a^{3}+27 b^{2}=x_{4} h$ for some polynomial $h$. We add $x_{5}$ times the first row of $M$ to the second row. We then divide all but the first row by $x_{4}$. Let $I \subset \mathbb{Q}\left[U, x_{1}, x_{2}, x_{3}, x_{4}, x_{5}\right]$ be the ideal generated by the $4 \times 4$ minors of $M$, and 


$$
J=\left\{f \in \mathbb{Q}\left[U, x_{1}, x_{2}, x_{3}, x_{4}, x_{5}\right]: x_{2} h f \in I\right\} .
$$

Using the Gröbner basis machinery in Magma we find that $J \cap \mathbb{Q}\left[U, x_{1}, x_{2}, x_{3}, x_{4}\right]$ is generated by 3 homogeneous polynomials of degree 4 . These define a surface in $\mathbb{P}^{4}$ of degree 12. By the substitution

$$
T=\frac{4\left(2 x_{1}-x_{2}+x_{3}\right) U+\left(x_{1} x_{2}-x_{2}^{2}+x_{3} x_{4}\right)}{2\left(2 x_{4}-x_{2}\right) U+2\left(x_{1} x_{2}-x_{2}^{2}+x_{3} x_{4}\right)}
$$

this surface is birational to the surface $\left\{Q_{1}=Q_{2}=0\right\}$ in $\mathbb{A}^{1} \times \mathbb{P}^{3}$ where

$$
\begin{aligned}
Q_{1}= & 4(T-2) x_{1} x_{2}+8 x_{1} x_{3}+(T-2)^{2} x_{2}^{2} \\
& +4(T-2) x_{2} x_{3}+2\left(T^{2}-T+1\right) x_{2} x_{4}+4 x_{3}^{2}-4 T x_{3} x_{4}, \\
Q_{2}= & 8 x_{1}^{2}+16 x_{1} x_{3}-4(2 T-1) x_{1} x_{4}-T(T-2) x_{2}^{2}-T^{2} x_{2} x_{3} \\
& -2\left(T^{2}-T+1\right) x_{2} x_{4}-2(T-4) x_{3}^{2}-2\left(T^{2}+4 T-1\right) x_{3} x_{4} .
\end{aligned}
$$

These same equations define a genus one curve in $\mathbb{P}^{3}$ defined over $\mathbb{Q}(T)$, with a rational point at $\left(x_{1}: x_{2}: x_{3}: x_{4}\right)=(0: 0: 0: 1)$. Putting this elliptic curve in Weierstrass form gives the equation in the statement of Theorem 1.2.

\section{Case $(N, \varepsilon)=(12,1)$}

Let $E$ be the elliptic curve $y^{2}=x^{3}+a x+b$. Equations for $X_{E}(12,1)$ as a curve in $\mathbb{P}^{5}$ were computed in [7, Theorem 1.7.10]. These equations are $F_{0}=F_{1}=F_{2}=F_{3}=0$ where

$$
\begin{aligned}
& F_{0}=-X^{2} Z+a X Y^{2}+6 b Y^{3}-6 a Y^{2} Z-12 Z^{3}, \\
& F_{1}=X^{2}+12 X Z+36 Z^{2}-2 u_{0} u_{2}-u_{1}^{2}+a u_{2}^{2}, \\
& F_{2}=4 a X Y+36 b Y^{2}-24 a Y Z-2 u_{0} u_{1}+2 a u_{1} u_{2}+b u_{2}^{2}, \\
& F_{3}=8 a X Z-4 a^{2} Y^{2}-u_{0}^{2}+2 b u_{1} u_{2} .
\end{aligned}
$$

These polynomials satisfy

$$
F_{i}\left(\lambda^{2} a, \lambda^{3} b ; \lambda X, Y, \lambda Z, \lambda^{2} u_{0}, \lambda u_{1}, u_{2}\right)=\lambda^{m_{i}} F_{i}\left(a, b ; X, Y, Z, u_{0}, u_{1}, u_{2}\right)
$$

where $\left(m_{0}, m_{1}, m_{2}, m_{3}\right)=(3,2,3,4)$. Again, it is our aim to quotient out by this $\mathbb{G}_{m}$-action. We do this by setting $(X+6 Z) Y=u_{2}^{2}$, which works since the left hand side and right hand side scale by consecutive powers of $\lambda$. Specifically, we substitute $\left(X, Y, Z, u_{0}, u_{1}, u_{2}\right)=$ $\left(x^{2}-6 y, 1, y, v x, w x, x\right)$ and then solve for $a$ and $b$ so that the first two equations are satisfied. In the remaining two equations we substitute

$$
v=2(w-2 y) y+\frac{T+1}{T-1}\left(x^{2}(y+1)-(w+2 y)^{2}\right) .
$$

The resultant of these two equations with respect to $w$ is $f(T) x^{14} y^{2} g(x, y)^{2} h(x, \tilde{y})$ where $f(T)$ is a rational function in $T, g(x, y)=x^{6}(y+1)-9 y^{2}\left(x^{2}+4\right)^{2}$,

$$
\begin{aligned}
h(x, y)= & (T+1)^{2} x^{2} y^{2}+(T+2)\left(T^{2}+3\right)^{2} x^{2} \\
& -4(T-1)(T+3)^{2} x y+4(T+3)^{2} y^{2}+12 T(T+1)^{2}(T+3)^{2}
\end{aligned}
$$

and $\tilde{y}=\left(144(T+1) y+(T+3)^{2}\left((T-3) x^{2}+12(T+1)\right)\right) /(8(T+3) x)$. Therefore $Z(12,1)$ is birational to the total space for the genus one curve $C=\{h=0\}$ in $\mathbb{A}^{2}$ defined 
over $\mathbb{Q}(T)$. Replacing $x$ by $2(T+3) /\left(T^{2}+3\right) x$, and completing the square in $y$ shows that $C$ has equation

$$
Y^{2}=-(T+2) x^{4}-\left(4 T^{3}+5 T^{2}+6 T+9\right) x^{2}-3 T\left(T^{2}+3\right)^{2} .
$$

This gives a genus one fibration on $Z(12,1)$ defined over $\mathbb{Q}$, but without a $\mathbb{Q}$-rational section. Indeed the fibres with $T>0$ have no real points.

We now find another genus one fibration that does have a $\mathbb{Q}$-rational section. Let $F\left(x_{1}, x_{2}, x_{3}\right)$ be the unique homogeneous polynomial of degree 6 with the property that $F(x, T, 1)$ is the right hand side of (1). Then $F$ is the discriminant of the following quadratic in $T$.

$$
x_{1}^{2} x_{2}+\left(T^{2}+2\right) x_{1}^{2} x_{3}+2 T x_{1} x_{2}^{2}-2 T x_{1} x_{2} x_{3}+T^{2} x_{2}^{3}+3 x_{2}^{2} x_{3}+3 T^{2} x_{2} x_{3}^{2}+9 x_{3}^{3}=0
$$

This same equation defines a genus one curve in $\mathbb{P}^{2}$ defined over $\mathbb{Q}(T)$, with a rational point at $\left(x_{1}: x_{2}: x_{3}\right)=(1: 0: 0)$. Putting this elliptic curve in Weierstrass form gives the equation in the statement of Theorem 1.1.

\section{Degree 3 covers of K3-surfaces}

We prove Theorems 1.1 and 1.2 in the cases $(N, \varepsilon)=(6,5),(10,1)$ and $(10,3)$. In the first of these cases, Chen's equations for $X_{E}(6,5)$ already give a genus one fibration on $Z(6,5)$, but one without a section. The content of Theorem 1.1 in this case is that we have found another genus one fibration that does have a section.

For $N$ an odd integer, let $Z^{*}(N, \varepsilon)$ be the double cover of $Z(N, \varepsilon)$ that parametrises pairs of elliptic curves that are $N$-congruent with power $\varepsilon$, and additionally have the property that their ratio of discriminants is a square.

Theorem 3.1 If $(N, \varepsilon)=(3,2)$, $(5,1)$ or $(5,2)$ then $Z^{*}(N, \varepsilon)$ is a double cover of $\mathbb{P}^{2}$, ramified over the union of two cuspidal cubics, with equation

$$
y^{2}=F_{+}(u, v, w) F_{-}(u, v, w)
$$

where

$$
\begin{array}{ll}
Z^{*}(3,2): & F_{ \pm}=u(u+3 v \pm w)^{2}+4 v^{3}, \\
Z^{*}(5,1): & F_{ \pm}=u\left(u^{2}-11 u v-v^{2}\right)+w^{2}(12 u+v) \pm 2 w\left(3 u^{2}-4 u v+4 w^{2}\right), \\
Z^{*}(5,2): & F_{ \pm}=u^{2}(11 v+8 w)+w^{2}(8 u-v+4 w) \pm 2 u(2 v-w)(4 u-v+4 w) .
\end{array}
$$

In particular, in each of these cases $Z^{*}(N, \varepsilon)$ is a K3-surface.

Proof Let $E$ be the elliptic curve $y^{2}=x^{3}+a x+b$. We put $\Delta=-4 a^{3}-27 b^{2}$, and define polynomials

$$
\begin{aligned}
& f(x)=x^{3}+a x+b, \\
& g(x)=3 a x^{4}+18 b x^{3}-6 a^{2} x^{2}-6 a b x-a^{3}-9 b^{2}, \\
& h(x)=3 a x^{2}+9 b x-a^{2}, \\
& j(x)=27 b x^{3}-18 a^{2} x^{2}-27 a b x-2 a^{3}-27 b^{2} .
\end{aligned}
$$

If we assign the variables $x, a, b$ weights $1,2,3$, then each of these polynomials is homogeneous. We note that $j^{2}=-4 h^{3}-27 \Delta f^{2}$. 
Case $(N, \boldsymbol{\varepsilon})=(3,2)$. The following equations for the family of curves parametrised by $X_{E}(3,2)$ are taken from [11, Section 13]. Starting from the Klein form ${ }^{1}$

$$
D(\xi, \eta)=-27 a \xi^{4}-54 b \xi^{3} \eta-18 a^{2} \xi^{2} \eta^{2}-54 a b \xi \eta^{3}+\left(a^{3}-27 b^{2}\right) \eta^{4},
$$

we define

$$
A(\xi, \eta)=\frac{1}{108}\left|\begin{array}{cc}
D_{\xi \xi} & D_{\xi \eta} \\
D_{\eta \xi} & D_{\eta \eta}
\end{array}\right|, \quad \text { and } \quad B(\xi, \eta)=\frac{1}{36}\left|\begin{array}{cc}
D_{\xi} & D_{\eta} \\
A_{\xi} & A_{\eta}
\end{array}\right|,
$$

where the subscripts denote partial derivatives. These forms satisfy the syzygy

$$
-4 A^{3}-27 B^{2}=16\left(4 a^{3}+27 b^{2}\right)^{2} D^{3} .
$$

The family of elliptic curves 3-congruent to $E$ with power $\varepsilon=2$ is given by

$$
y^{2}=x^{3}+A(\xi, \eta) x+B(\xi, \eta) .
$$

We dehomogenise by putting $(\xi, \eta)=(x, 1)$. Then $D=f_{x}^{3}-27 f^{2}=j-3 f_{x} h$ where $f_{x}=3 x^{2}+a$. The quantities $(u, v, r, s)=\left(D, f_{x} h, h^{3}, 3^{6} \Delta f^{4}\right)$ are related by

$$
\left(4 r+(u+3 v)^{2}\right)\left(r u-v^{3}\right)=r s .
$$

As we verify in Remark 3.2 below, this is an equation for $Z(3,2)$ in $\mathbb{P}(1,1,2,3)$ where the coordinates $u, v, r, s$ have weights $1,1,2,3$. We see by (3) that, up to squared factors, the ratio of discriminants is $s / u$. We substitute $s=u w^{2}$ in (4) to give a quadratic in $r$ whose discriminant is the polynomial $F_{+} F_{-}$in the statement of the theorem.

Case $(\boldsymbol{N}, \boldsymbol{\varepsilon})=(\mathbf{5}, \mathbf{1})$. The following equations for the family of curves parametrised by $X_{E}(5,1)$ are taken from [11, Section 13]. Starting from the Klein form ${ }^{2}$

$$
\begin{aligned}
D(\lambda, \mu)= & \lambda^{12}+22 a \lambda^{10} \mu^{2}+220 b \lambda^{9} \mu^{3}-165 a^{2} \lambda^{8} \mu^{4}-528 a b \lambda^{7} \mu^{5} \\
& -220\left(a^{3}+12 b^{2}\right) \lambda^{6} \mu^{6}+264 a^{2} b \lambda^{5} \mu^{7}-165 a\left(5 a^{3}+32 b^{2}\right) \lambda^{4} \mu^{8} \\
& -880 b\left(3 a^{3}+20 b^{2}\right) \lambda^{3} \mu^{9}+22 a^{2}\left(25 a^{3}+168 b^{2}\right) \lambda^{2} \mu^{10} \\
& +20\left(19 a^{4} b+128 a b^{3}\right) \lambda \mu^{11}+\left(125 a^{6}+1792 a^{3} b^{2}+6400 b^{4}\right) \mu^{12},
\end{aligned}
$$

we define

$$
A(\lambda, \mu)=\frac{1}{5808}\left|\begin{array}{cc}
D_{\lambda \lambda} & D_{\lambda \mu} \\
D_{\mu \lambda} & D_{\mu \mu}
\end{array}\right|, \quad \text { and } \quad B(\lambda, \mu)=\frac{1}{360}\left|\begin{array}{cc}
D_{\lambda} & D_{\mu} \\
A_{\lambda} & A_{\mu}
\end{array}\right|,
$$

where the subscripts denote partial derivatives. These forms satisfy the syzygy

$$
4 A^{3}+27 B^{2}=\left(4 a^{3}+27 b^{2}\right) D^{5} .
$$

The family of elliptic curves 5-congruent to $E$ with power $\varepsilon=1$ is given by

$$
y^{2}=x^{3}+A(\lambda, \mu) x+B(\lambda, \mu) .
$$

We dehomogenise by putting $(\lambda, \mu)=(x, 1)$. Then

$$
D=4 k f-3\left(f^{2}+g\right)^{2}+32 \Delta\left(f^{2}+g\right),
$$

${ }^{1}$ We obtain $D$ from $\mathfrak{D}(\xi, \eta)$ in [11, Section 9] by putting $c_{4}=-48 a, c_{6}=-864 b$, multiplying $\xi$ by 12 , and dividing through by $2^{12} 3^{3}$.

${ }^{2}$ We obtain $D$ from $\mathbf{D}(\lambda, \mu)$ in [11, Section 8] by putting $c_{4}=-48 a, c_{6}=-864 b$, multiplying $\lambda$ by 12 , and dividing through by $2^{24} 3^{12}$. 
where $k(x)=f^{3}+f j+4 \Delta f+3 g\left(x f_{x}-2 f\right)=x^{9}+12 a x^{7}+84 b x^{6}+\cdots$

The quantities $(t, u, v, r, s)=\left(4 f, 2\left(f^{2}+g\right), 16 \Delta, 4 k, D\right)$ are related by

$$
\begin{aligned}
r^{2}+s t^{2} & =u\left(u^{2}-11 u v-v^{2}\right)+(12 u+v) s, \\
r t & =3 u^{2}-4 u v+4 s .
\end{aligned}
$$

These are equations for $Z(5,1)$ in $\mathbb{P}(1,2,2,3,4)$ where the coordinates $t, u, v, r, s$ have weights $1,2,2,3,4$. We see by (5) that, up to squared factors, the ratio of discriminants is $s$. Putting $s=w^{2}$ we obtain from (6) the equation

$$
\left(r^{2}-s t^{2}\right)^{2}=\left(r^{2}+s t^{2}\right)^{2}-4 s(r t)^{2}=F_{+}(u, v, w) F_{-}(u, v, w)
$$

where $F_{ \pm}$are the polynomials in the statement of the theorem.

Case $(N, \varepsilon)=(5,2)$. The following equations for the family of curves parametrised by $X_{E}(5,2)$ are taken from [12, Theorem 5.8]. Starting from the Klein form ${ }^{3}$

$$
\begin{aligned}
D(\lambda, \mu)= & \left(125 a^{3}-432 b^{2}\right) \lambda^{12}+2430 a^{2} b \lambda^{11} \mu-22 a\left(25 a^{3}-378 b^{2}\right) \lambda^{10} \mu^{2} \\
& -110 b\left(11 a^{3}-108 b^{2}\right) \lambda^{9} \mu^{3}-165 a^{2}\left(5 a^{3}-27 b^{2}\right) \lambda^{8} \mu^{4}-132 a b\left(53 a^{3}-189 b^{2}\right) \lambda^{7} \mu^{5} \\
& +220\left(a^{6}-123 a^{3} b^{2}+81 b^{4}\right) \lambda^{6} \mu^{6}+132 a^{2} b\left(19 a^{3}-297 b^{2}\right) \lambda^{5} \mu^{7} \\
& -165\left(a^{7}-26 a^{4} b^{2}+189 a b^{4}\right) \lambda^{4} \mu^{8}-110\left(3 a^{6} b-34 a^{3} b^{3}+135 b^{5}\right) \lambda^{3} \mu^{9} \\
& -22 a^{2}\left(a^{3}-3 b^{2}\right)\left(a^{3}+27 b^{2}\right) \lambda^{2} \mu^{10}-10 a b\left(5 a^{6}+82 a^{3} b^{2}+189 b^{4}\right) \lambda \mu^{11} \\
& +\left(a^{9}-a^{6} b^{2}-181 a^{3} b^{4}-675 b^{6}\right) \mu^{12},
\end{aligned}
$$

we define

$$
A(\lambda, \mu)=\frac{1}{1452}\left|\begin{array}{ll}
D_{\lambda \lambda} & D_{\lambda \mu} \\
D_{\mu \lambda} & D_{\mu \mu}
\end{array}\right|, \quad \text { and } B(\lambda, \mu)=\frac{-1}{180}\left|\begin{array}{cc}
D_{\lambda} & D_{\mu} \\
A_{\lambda} & A_{\mu}
\end{array}\right|,
$$

where the subscripts denote partial derivatives. These forms satisfy the syzygy

$$
-4 A^{3}-27 B^{2}=16\left(4 a^{3}+27 b^{2}\right)^{2} D^{5} .
$$

The family of elliptic curves 5-congruent to $E$ with power $\varepsilon=2$ is given by

$$
y^{2}=x^{3}+A(\lambda, \mu) x+B(\lambda, \mu) .
$$

We dehomogenise by putting $(\lambda, \mu)=(x, 1)$. Then

$$
D=16 \Delta f^{4}-g^{3}+4\left(2 g^{3}-g^{2} j-4 \Delta f^{2} g\right) .
$$

The quantities $(r, s, v, w)=\left(\Delta f^{4}, \Delta f^{2} g, g^{3}, 2 g^{3}-g^{2} j-4 \Delta f^{2} g\right)$ are related by

$$
r(4 s-2 v+w)^{2}+27 r s v+s w^{2}-s^{2}(v-4 w)=0 .
$$

This is an equation for $Z(5,2)$ as a cubic surface in $\mathbb{P}^{3}$. We see from (7) and (8) that, up to squared factors, the ratio of discriminants is $r(16 r-v+4 w)$. Putting $r(16 r-v+4 w)=$ $(4 r-u)^{2}$, where $u$ is a new variable, and using this equation to eliminate $r$ from (9), we obtain a quadratic in $s$ whose discriminant is the polynomial $F_{+} F_{-}$in the statement of the theorem.

For the last part of Theorem 3.1, we note that one of the standard constructions of a K3surface is as a double cover of $\mathbb{P}^{2}$ ramified over a non-singular sextic curve. In our case the

\footnotetext{
${ }^{3}$ We obtain $D$ from $\mathbf{D}(\lambda, \mu)$ in [12, Section 5] by putting $c_{4}=-48 a, c_{6}=-864 b$, multiplying $\lambda$ by 12 , and dividing through by $2^{36} 3^{15}$.
} 
sextic is singular, but the surface is still (birational to) a K3-surface. See for example [18, Chapter 1, Example 1.3(iv)].

Remark 3.2 Let $(N, \varepsilon)=(3,2),(5,1)$ or $(5,2)$. We saw in the proof of Theorem 3.1 that one model for $Z(N, \varepsilon)$ is the weighted projective plane $\mathbb{P}(1,2,3)$ where the co-ordinates $x, a, b$ have weights $1,2,3$. We mapped this to another model for $Z(N, \varepsilon)$ defined by (4), (6) or (9). The inverse maps are as follows.

$$
\begin{aligned}
& \left\{\begin{array}{l}
x=r+v^{2} \\
a=-3 r\left(r+u v+2 v^{2}\right) \\
b=r(u+3 v)\left(r u+v^{3}\right)+2 r^{2}\left(r+3 v^{2}\right)
\end{array}\right. \\
& \left\{\begin{array}{l}
x=32 u-v+5 t^{2} \\
a=-3(8 u-v)(32 u-v)-288 r t+30(28 u+v) t^{2}-75 t^{4} \\
b=-2(32 u-v)^{2}(4 u+v)-144(32 u-v) r t+6(32 u-v)(88 u-5 v) t^{2}
\end{array}\right. \\
& \left\{\begin{aligned}
& x=4 r s+4 r v+r w-s^{2} \\
& a=3\left(8 r s^{3}+4 r s^{2} v+6 r s^{2} w+r s w^{2}-s^{4}\right), \\
& b=r^{2} s\left(16 s^{3}-8 s^{2} v-24 s^{2} w-40 s v w-15 s w^{2}+4 v w^{2}-2 w^{3}\right) \\
& \quad+r s^{3}\left(24 s^{2}+8 s v+34 s w+7 w^{2}\right)-2 s^{6} .
\end{aligned}\right.
\end{aligned}
$$

Remark 3.3 There are two naturally defined involutions on the K3-surfaces in Theorem 3.1. The first switches the sign of $y$, and corresponds to swapping over the pair of $N$-congruent elliptic curves. The second is given on $Z^{*}(3,1)$ and $Z^{*}(5,1)$ by switching the sign of $w$, and on $Z^{*}(5,2)$ by $(u, v, w, y) \mapsto\left(\tilde{u}, v, w,(\tilde{u} / u)^{2} y\right)$ where $\tilde{u}=u(v-4 w) /(8 u-(v-4 w))$. This second involution switches the choice of square root for the ratio of discriminants. The two involutions commute, and the second swaps over the curves $F_{+}=0$ and $F_{-}=0$.

Remark 3.4 For a suitable parametrisation of the cuspidal cubic $F_{+}=0$, we obtain a family of elliptic curves with $j$-invariant

$$
\begin{array}{ll}
(3,1): & j=27(T-3)^{3}(T+1)^{3} / T^{3}, \\
(5,1): & j=(T+5)^{3}\left(T^{2}-5\right)^{3}\left(T^{2}+5 T+10\right)^{3} /\left(T^{2}+5 T+5\right)^{5}, \\
(5,2): & j=125 T(2 T+1)^{3}\left(2 T^{2}+7 T+8\right)^{3} /\left(T^{2}+T-1\right)^{5} .
\end{array}
$$

These correspond to $X_{\mathrm{s}}^{+}(3), X_{\mathrm{s}}^{+}(5)$ and $X_{\mathrm{ns}}^{+}(5)$, where $X_{\mathrm{s}}^{+}(N)$ and $X_{\mathrm{ns}}^{+}(N)$ are the modular curves associated to the normaliser of a split or non-split Cartan subgroup of level $N$. We may compute $X_{\mathrm{s}}^{+}(N)$ as the quotient of $X_{0}\left(N^{2}\right)$ by the Fricke involution, whereas the formula for $X_{\mathrm{ns}}^{+}(5)$ is taken from [6, Corollary 5.3]. The use of these modular curves to construct pairs of $N$-congruent elliptic curves is described further in [16].

Let $N$ be an odd integer and let $\varepsilon \in(\mathbb{Z} / 2 N \mathbb{Z})^{\times}$. Then $X(2 N) \rightarrow X(N)$, and hence also $X_{E}(2 N, \varepsilon) \rightarrow X_{E}(N, \varepsilon)$, is geometrically a Galois covering with Galois group $\mathrm{PSL}_{2}(\mathbb{Z} / 2 \mathbb{Z}) \cong S_{3}$. Since the discriminant of an elliptic curve is equal (up to a constant factor) to the discriminant of its 2-division polynomial, elliptic curves which are 2-congruent have ratio of discriminants a square. It follows that $Z(2 N, \varepsilon) \rightarrow Z^{*}(N, \varepsilon)$ is a degree 3 cover. In the cases $(2 N, \varepsilon)=(6,5),(10,1)$ and $(10,3)$ it turns out that the surface $Z(2 N, \varepsilon)$ 
has an elliptic fibration. The pushforward of a fibre gives a divisor class $D$ on the K3-surface $Z^{*}(N, \varepsilon)$ with $D^{2}=2$. Using this divisor class $D$ we may write $Z^{*}(N, \varepsilon)$ as a double cover of $\mathbb{P}^{2}$. We have arranged (with the benefit of hindsight) that the equations in Theorem 3.1 write $Z^{*}(N, \varepsilon)$ as a double cover of $\mathbb{P}^{2}$ in exactly this way.

The equations for $Z(2 N, \varepsilon)$ in Theorems 1.1 and 1.2 may be obtained from the equations for $Z^{*}(N, \varepsilon)$ in Theorem 3.1 as follows. The tangent line to a general point on the cuspidal cubic $F_{+}(u, v, w)=0$ has equation:

$$
\begin{aligned}
& (2 N, \varepsilon)=(6,5) \\
& \left(T^{3}-1\right) u+3(T-1) v-w=0, \\
& (2 N, \varepsilon)=(10,1) \\
& (2 N, \varepsilon)=(10,3) \\
& (T-2) u-T(T-1)^{2} v+2(T-1) w=0, \\
& T^{3} u-(T+1) v-T^{2} w=0 .
\end{aligned}
$$

We parametrise this line, and substitute into the right hand side of the equation $y^{2}=$ $F_{+}(u, v, w) F_{-}(u, v, w)$. After cancelling a squared factor (which arises since we chose a tangent line) the right hand side is a binary quartic with a linear factor. We now have the equation for a genus one curve over $\mathbb{Q}(T)$ with a rational point. Putting this elliptic curve in Weierstrass form gives the equations for $Z(6,5), Z(10,1)$ and $Z(10,3)$ in Theorems 1.1 and 1.2.

It remains to show that the degree 3 covers of $Z^{*}(N, \varepsilon)$ defined by $(10),(11)$ and (12) are indeed birational to $Z(2 N, \varepsilon)$. To do this we must check that a point on $Z^{*}(N, \varepsilon)$ lifts to the degree 3 cover precisely when the corresponding pair of elliptic curves are 2 -congruent. We use the following lemma.

Lemma 3.5 Let $K$ be a field of characteristic not 2 or 3 . Elliptic curves $E_{1}$ and $E_{2}$ over $K$ with $j$-invariants $j_{1}$ and $j_{2}$, with $j_{1}, j_{2} \notin\{0,1728\}$, are 2-congruent if and only if there exist $m, x \in K$ satisfying $\left(j_{1}-1728\right)\left(j_{2}-1728\right)=m^{2}$ and

$$
x^{3}-3 j_{1} j_{2} x-2 j_{1} j_{2}(m+1728)=0 .
$$

Proof This follows from the formulae in [28] or [11, Sections 8 and 13] by a generic calculation.

We illustrate the use of Lemma 3.5 in the case $(2 N, \varepsilon)=(10,3)$, the other cases being similar, and treated in the accompanying Magma file. Above each point $(u: v: w) \in \mathbb{P}^{2}$ there are a pair of points on $Z^{*}(5,2)$ possibly defined over a quadratic extension. These points correspond to a pair of elliptic curves, say with $j$-invariants $j_{1}$ and $j_{2}$. A calculation using the formulae in Remark 3.2 shows that, for $m$ a suitable choice of square root of $\left(j_{1}-1728\right)\left(j_{2}-1728\right)$, we have

$$
\begin{aligned}
j_{1} j_{2} & =G_{6}(u, v, w) H(u, v, w)^{2} \\
j_{1} j_{2}(m+1728) & =G_{9}(u, v, w) H(u, v, w)^{3}
\end{aligned}
$$

where

$$
\begin{aligned}
& G_{6}(u, v, w)=640 u^{4} v^{2}-768 u^{4} v w-72 u^{3} v^{3}-240 u^{3} v^{2} w+\cdots \\
& G_{9}(u, v, w)=6912 u^{7} v^{2}-1376 u^{6} v^{3}-14976 u^{6} v^{2} w+\cdots
\end{aligned}
$$

are irreducible homogeneous polynomials of degrees 6 and 9 , and $H \in \mathbb{Q}(u, v, w)$ is a rational function. Finally we claim that the polynomials

$$
X^{3}-3 G_{6}(u, v, w) X-2 G_{9}(u, v, w)=0,
$$


arising from Lemma 3.5, and

$$
u T^{3}-w T^{2}-v T-v=0
$$

appearing in (12), define the same cubic extension. Indeed we find by computer algebra that if (14) has root $T_{0}$ then (13) has root

$$
\begin{aligned}
X_{0}= & 3 u^{2}(8 u-3 v-4 w) T_{0}^{2}+12 u(2 u v-4 u w+v w) T_{0} \\
& -16 u^{2} v+6 u v^{2}+8 u w^{2}-v w^{2}+4 w^{3} .
\end{aligned}
$$

\section{Computing the Picard numbers}

Let $E / \mathbb{Q}(T)$ be one of the elliptic curves in Theorems 1.1 and 1.2. We write $X \rightarrow \mathbb{P}^{1}$ for the minimal fibred surface with generic fibre $E$. The reduction of $E \bmod p$ is an elliptic curve $E_{p} / \mathbb{F}_{p}(T)$, and the reduction of $X \bmod p$ is a surface $X_{p} / \mathbb{F}_{p}$. We will always take $p$ to be a prime of good reduction.

Let $\bar{X}=X \times \mathbb{Q} \overline{\mathbb{Q}}$ and $\bar{X}_{p}=X_{p} \times_{\mathbb{F}_{p}} \overline{\mathbb{F}}_{p}$. We write NS for the Néron-Severi group. The Shioda-Tate formula [29, Corollary 5.3] tells us that

$$
\operatorname{rank} E(\overline{\mathbb{Q}}(T))+2+\sum_{t \in \mathbb{P}^{1}(\overline{\mathbb{Q}})}\left(m_{t}-1\right)=\operatorname{rank} \mathrm{NS}(\bar{X}),
$$

and

$$
\operatorname{rank} E_{p}\left(\overline{\mathbb{F}}_{p}(T)\right)+2+\sum_{t \in \mathbb{P}^{1}\left(\overline{\mathbb{F}}_{p}\right)}\left(m_{t}-1\right)=\operatorname{rank} \operatorname{NS}\left(\bar{X}_{p}\right),
$$

where $m_{t}$ is the number of irreducible components in the fibre above $t$. We write $\rho$ and $\rho_{p}$ for the numbers on the right of (15) and (16). These are the geometric Picard numbers of $X$ and $X_{p}$. The sections exhibited in Tables 3 and 4 give a lower bound for rank $E(\overline{\mathbb{Q}}(T))$ and hence by (15) a lower bound for $\rho$. These lower bounds are exactly the values recorded in Tables 1 and 2.

Let $X \rightarrow \mathbb{P}^{1}$ be a minimal elliptic surface with non-constant $j$-invariant, and let $m=$ $\chi\left(\mathcal{O}_{X}\right)$ be the Euler characteristic of the structure sheaf. This may be computed from the fact [22, III.4.4 and IV.3.3] that the sum of the Euler numbers of the singular fibres is $12 \mathrm{~m}$. By [22, Lemma IV.1.1] the Hodge diamond of $X$ is

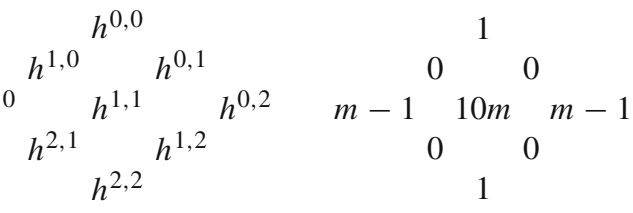

The surfaces in Theorem 1.1 have $m=2$ and those in Theorem 1.2 have $m=3$. This may be checked by computing the Euler numbers, but more simply corresponds to the fact that $m$ is the least integer such that the coefficients $a_{1}, \ldots, a_{6}$ of the Weierstrass equations satisfy $\operatorname{deg}\left(a_{i}\right) \leq m i$ for all $i$. To tie in with [19, Theorem 4], we note that $p_{g}=h^{2,0}=m-1$. By the Lefschetz theorem on $(1,1)$-classes (see for example [2, Chapter IV, Theorem 2.13]) we have $\rho \leq h^{1,1}=10 \mathrm{~m}$. This already determines $\rho$ in all cases with $N \leq 8$. It remains for us to improve this upper bound by 1 in the cases $(N, \varepsilon)=(9,1),(12,1),(9,2)$, and to improve it by 2 in the cases $(N, \varepsilon)=(10,1),(10,3),(11,1)$.

The main tool we wish to use (see [33, Proposition 6.2]) is that there is an injective map $\mathrm{NS}(\bar{X}) \rightarrow \mathrm{NS}\left(\bar{X}_{p}\right)$ that preserves the intersection pairing. 
Table 5 Bounding the geometric Picard number

\begin{tabular}{llll}
\hline$(N, \varepsilon)$ & Characteristic polynomial of Frobenius $f_{p}(x)$ & $\operatorname{deg} g_{p}$ & $\Delta_{p}$ \\
\hline$(9,1)$ & $f_{5}(x)=(x-1)^{16}(x+1)^{2}\left(x^{2}+x+1\right)\left(x^{2}+\frac{7}{5} x+1\right)$ & 20 & $3 \cdot 17$ \\
& $f_{7}(x)=(x-1)^{18}(x+1)^{2}\left(x^{2}+\frac{10}{7} x+1\right)$ & 20 & $2 \cdot 3$ \\
$(12,1)$ & $f_{5}(x)=(x-1)^{16}(x+1)^{4}\left(x^{2}+\frac{6}{5} x+1\right)$ & 20 & 1 \\
& $f_{11}(x)=(x-1)^{12}(x+1)^{8}\left(x^{2}+\frac{6}{11} x+1\right)$ & 20 & 7 \\
$(9,2)$ & $f_{7}(x)=(x-1)^{24}(x+1)^{2}\left(x^{2}+x+1\right)^{2}\left(x^{2}+\frac{10}{7} x+1\right)\left(x^{2}+\frac{13}{7} x+1\right)$ & 30 & 2 \\
& $f_{13}(x)=(x-1)^{24}\left(x^{2}+x+1\right)^{3}\left(x^{2}+\frac{1}{13} x+1\right)\left(x^{2}+\frac{25}{13} x+1\right)$ & 30 & 17 \\
$(10,1) \quad$ & $f_{7}(x)=(x-1)^{24}(x+1)^{2}\left(x^{2}+x+1\right)^{2}\left(x^{2}+\frac{10}{7} x+1\right)^{2}$ & 30 & 1 \\
& $f_{17}(x)=-(x-1)^{25}(x+1)^{5}\left(x^{2}-\frac{2}{17} x+1\right)\left(x^{2}+\frac{25}{17} x+1\right)$ & 30 & $2 \cdot 59$ \\
$(10,3)$ & $f_{31}(x)=(x-1)^{24}(x+1)^{2}\left(x^{2}+x+1\right)^{2}\left(x^{2}+\frac{46}{31} x+1\right)\left(x^{2}+\frac{58}{31} x+1\right)$ & 30 & $2 \cdot 5$ \\
& $f_{37}(x)=(x-1)^{28}(x+1)^{2}\left(x^{2}+\frac{70}{37} x+1\right)^{2}$ & 30 & 1 \\
$(11,1)$ & $f_{23}(x)=(x-1)^{28}(x+1)^{2}\left(x^{2}+\frac{42}{23} x+1\right)\left(x^{2}+\frac{45}{23} x+1\right)$ & 30 & $2 \cdot 7 \cdot 11 \cdot 13$ \\
& $f_{53}(x)=(x-1)^{28}\left(x^{2}+x+1\right)\left(x^{2}+\frac{25}{53} x+1\right)\left(x^{2}+\frac{70}{53} x+1\right)$ & 30 & $11 \cdot 131$ \\
\hline
\end{tabular}

Let $f_{p}(x)$ be the characteristic polynomial of Frobenius acting on $H_{e t}^{2}\left(\bar{X}_{p}, \mathbb{Q}_{\ell}(1)\right)$, normalised so that $f_{p}(0)=1$. This is a polynomial of degree $b_{2}=12 m-2$, independent of the choice of prime $\ell \neq p$. By the Weil conjectures it satisfies the functional equation $f_{p}(x)= \pm x^{b_{2}} f_{p}(1 / x)$. The polynomials $f_{p}(x)$ may be computed from the numbers $n_{r}=\left|X_{p}\left(\mathbb{F}_{p^{r}}\right)\right|$ using the Lefschetz trace formula. See for example [34, Section 3], where $f_{p}$ is denoted $\widetilde{f}_{p}$. We used both the functional equation and the Magma function FrobeniusActionOnTriviallattice to limit how many $n_{r}$ we had to compute. The polynomials $f_{p}(x)$ for two primes of good reduction (carefully chosen with the calculations at the end of this section in mind) are recorded in Table 5, and again in the accompanying Magma file.

Let $\Delta_{p} \in \mathbb{Q}^{\times} /\left(\mathbb{Q}^{\times}\right)^{2}$ be the absolute value of the determinant of the intersection pairing on $\operatorname{NS}\left(\bar{X}_{p}\right)$. It may be computed using either of the following two lemmas. We emphasise at this point that our results in Tables 1 and 2 are unconditional. In particular they do not depend on any unproven cases of the Tate conjecture.

Lemma 4.1 Write $f_{p}(x)=g_{p}(x) h_{p}(x)$ where every root of $g_{p}$ is a root of unity, and no root of $h_{p}$ is a root of unity. Then $\rho_{p} \leq \operatorname{deg} g_{p}$ and in the case of equality we have $\Delta_{p} \equiv$ $h_{p}(1) h_{p}(-1) \bmod \left(\mathbb{Q}^{\times}\right)^{2}$.

Proof. The first part is described for example in [34, Corollary 2.3]. The Tate conjecture predicts that this inequality is always an equality, and this has been proved for K3-surfaces. See [18, Section 17.3] or [32] for the history of this problem and further references. The formula for $\Delta_{p}$ is a small refinement of a result of Kloosterman, that in turn depends on known cases of the Artin-Tate conjecture.

Let $F_{k}(x)=\prod\left(1-p^{k} \alpha_{i}^{k} x\right)$ where $f_{p}(x)=\prod\left(1-\alpha_{i} x\right)$. The result of Kloosterman [20, Proposition 4.7], is that if $k$ is an even integer with $\alpha_{i}^{k}=1$ for all $\alpha_{i}$ which are roots of unity, then

$$
\Delta_{p}=\lim _{s \rightarrow 1} \frac{F_{k}\left(p^{-k s}\right)}{\left(1-p^{k(1-s)}\right)^{\rho}} .
$$


Let $H_{k}(x)=\prod\left(1-p^{k} \beta_{i}^{k} x\right)$ where $h_{p}(x)=\prod\left(1-\beta_{i} x\right)$. Then $F_{k}(x)=\left(1-p^{k} x\right)^{\rho} H_{k}(x)$, and (17) becomes

$$
\Delta_{p}=H_{k}\left(p^{-k}\right)=\prod_{i}\left(\beta_{i}^{k}-1\right)=h_{p}(1) h_{p}(-1) \prod_{\substack{d \mid k \\ d>2}} \prod_{i} \Phi_{d}\left(\beta_{i}\right)
$$

where $\Phi_{d}$ is the $d$ th cyclotomic polynomial. For $d>2$ we claim that $\prod_{i} \Phi_{d}\left(\beta_{i}\right)$ is a rational square. By the functional equation we have

$$
\beta_{1}, \ldots, \beta_{2 m}=\gamma_{1}, \ldots, \gamma_{m}, \gamma_{1}^{-1}, \ldots, \gamma_{m}^{-1} .
$$

Since $d>2$ we have $\Phi_{d}(x)=x^{\phi(d)} \Phi_{d}(1 / x)$ where $\phi(d)$ is even, say $\phi(d)=2 n$. Therefore $\gamma_{i}^{-n} \Phi_{d}\left(\gamma_{i}\right)=\gamma_{i}^{n} \Phi_{d}\left(\gamma_{i}^{-1}\right)$. It follows that $\prod_{i=1}^{m} \gamma_{i}^{-n} \Phi_{d}\left(\gamma_{i}\right) \in \mathbb{Q}$ and

$$
\prod_{i=1}^{2 m} \Phi_{d}\left(\beta_{i}\right)=\prod_{i=1}^{2 m} \beta_{i}^{-n} \Phi_{d}\left(\beta_{i}\right)=\left(\prod_{i=1}^{m} \gamma_{i}^{-n} \Phi_{d}\left(\gamma_{i}\right)\right)^{2} .
$$

Lemma 4.2 Suppose that $P_{1}, \ldots P_{r}$ generate a finite index subgroup of $E_{p}\left(\overline{\mathbb{F}}_{p}(T)\right)$. Then we have $\Delta_{p} \equiv\left(\prod_{t} c_{t}\right) \operatorname{Reg}\left(P_{1}, \ldots, P_{r}\right) \bmod \left(\mathbb{Q}^{\times}\right)^{2}$ where the product is over $t \in \mathbb{P}^{1}\left(\overline{\mathbb{F}}_{p}\right)$ and $c_{t}$ is the number of irreducible components of multiplicity one in the fibre of $\bar{X}_{p}$ above $t$.

Proof See [29, Theorem 8.7 and (7.8)].

In the calculations below, we sometimes needed to find explicit generators for $E_{p}\left(\mathbb{F}_{p}(T)\right)$. These were found by searching on 2-coverings, computed using 2-descent in Magma, as implemented in the function field case by S. Donnelly.

If $\rho=\rho_{p}=\rho_{q}$ for distinct primes $p$ and $q$, then (by the properties of the map $\operatorname{NS}(\bar{X}) \rightarrow$ NS $\left(\bar{X}_{p}\right)$ cited above) we have $\Delta_{p} \equiv \Delta_{q} \bmod \left(\mathbb{Q}^{\times}\right)^{2}$. As observed by van Luijk [34], this can sometimes be used to improve our upper bound on $\rho$ by 1 . This is particularly useful since (assuming the Tate conjecture) $\rho_{p}$ is always even. Indeed $\rho_{p}=\operatorname{deg} g_{p}=b_{2}-\operatorname{deg} h_{p}$, and $\operatorname{deg} h_{p}$ is even by the functional equation. See [20] and [23] for further examples.

Case $(\boldsymbol{N}, \boldsymbol{\varepsilon})=(\mathbf{9}, \mathbf{1})$. From the second and third paragraphs of this section we already know that $\rho=19$ or 20. Since the Tate conjecture has been proved for elliptic K3-surfaces [1], equality holds in Lemma 4.1. By Lemma 4.1 we compute $\Delta_{5}=3 \cdot 17$ and $\Delta_{7}=2 \cdot 3$, it being understood that these are elements of $\mathbb{Q}^{\times} /\left(\mathbb{Q}^{\times}\right)^{2}$. Since these are different, it follows by the method of van Luijk that $\rho=19$.

Case $(\boldsymbol{N}, \boldsymbol{\varepsilon})=(\mathbf{1 2}, \mathbf{1})$. This is identical to the previous example, except that now $\Delta_{5}=1$ and $\Delta_{11}=7$.

Case $(\boldsymbol{N}, \boldsymbol{\varepsilon})=(\mathbf{9}, \mathbf{2})$. We already know that $\rho=29$ or 30 . Let $p=7$ or 13 . By Lemma 4.1 and (16) we have rank $E_{p}\left(\overline{\mathbb{F}}_{p}(T)\right) \leq 3$. We prove equality by exhibiting three independent points of infinite order in $E_{p}\left(\mathbb{F}_{p}(T)\right)$. In addition to the reductions of the two points in Table 3, we have when $p=7$ the point

$$
\left(5 T^{5}+6 T^{4}+4 T^{2}+6 T, T^{7}+3 T^{6}+6 T^{3}+2 T^{2}+2 T\right),
$$

and when $p=13$ the point

$$
\left(4 T^{6}+8 T^{5}+3 T^{4}+7 T^{3}+5 T^{2}+10 T+2,10 T^{9}+4 T^{8}+5 T^{6}+5 T^{5}+12 T^{3}+4 T^{2}+12\right) .
$$


Using either Lemma 4.1 or Lemma 4.2 we find that $\Delta_{7}=2$ and $\Delta_{13}=17$. Since these are different, it follows that $\rho=29$. As the referee points out, had we assumed for a contradiction that $\rho=30$, then this would already give equality in Lemma 4.1 for $p=7$ and 13. Computing the extra points was therefore not necessary in this case.

In the cases $(N, \varepsilon)=(10,1),(10,3),(11,1)$ we aim to show that $\rho=28$. We were unable ${ }^{4}$ to find a prime $p$ with $\rho_{p}=28$, despite computing the polynomials $f_{p}(x)$ for all $p<150$. This prompted us to try a variant of van Luijk's method, where we use the intersection pairing to improve our upper bound for $\rho$ by 2 .

Case $(N, \boldsymbol{\varepsilon})=(\mathbf{1 0}, \mathbf{1})$. We already know that $\rho=28,29$ or 30 . In addition to the point $P_{1}=(0,0)$ in Table 3 we have when $p=7$ the points

$$
\begin{aligned}
& Q_{1}=\left(6 T^{6}+6 T^{4}+4 T^{3}+5 T^{2}, 4 T^{9}+6 T^{8}+6 T^{7}+T^{6}+T^{5}+3 T^{4}\right), \\
& Q_{2}=\left(T^{6}+5 T^{5}+6 T^{4}+4 T^{3}+5 T^{2}, 2 T^{9}+6 T^{8}+2 T^{7}+T^{6}+3 T^{4}\right),
\end{aligned}
$$

and when $p=17$ the points

$$
\begin{aligned}
& R_{1}=\left(16 T^{6}+13 T^{5}+6 T^{4}+4 T^{3}+12 T^{2}, 4 T^{9}+2 T^{8}+5 T^{7}+8 T^{5}+15 T^{4}\right), \\
& R_{2}=\left(\left(6 T^{8}+8 T^{7}+2 T^{6}+5 T^{5}+8 T^{4}+4 T^{3}+T^{2}\right) /(T+6)^{2}, \ldots\right) .
\end{aligned}
$$

We checked that these points are independent. Therefore by Lemma 4.1 and (16) we have $\rho_{p}=30$ and rank $E_{p}\left(\overline{\mathbb{F}}_{p}(T)\right)=3$ for $p=7$ and 17 . Using either Lemma 4.1 or Lemma 4.2 we find that $\Delta_{7}=1$ and $\Delta_{17}=2 \cdot 59$. Since these are different, it follows that $\rho \leq 29$.

As we checked using Magma, reducing mod 7 or 17 does not change the Kodaira symbols of the singular fibres. So by Lemma 4.2 it will be enough for us to work with the height pairing on the Mordell-Weil group, rather than the intersection pairing on the full Néron-Severi group. We compute

$$
\begin{aligned}
\operatorname{Reg}\left(P_{1}, u Q_{1}+v Q_{2}\right) & =\frac{2}{75}\left(7 u^{2}-12 u v+18 v^{2}\right), \\
\operatorname{Reg}\left(P_{1}, x R_{1}+y R_{2}\right) & =\frac{1}{450}\left(139 x^{2}+76 x y+316 y^{2}\right) .
\end{aligned}
$$

If $\rho=29$ then we have $\operatorname{rank} E(\overline{\mathbb{Q}}(T))=2$, and so there exists $P_{2} \in E(\overline{\mathbb{Q}}(T))$ whose reduction mod 7 is $\lambda P_{1}+u Q_{1}+v Q_{2}$ and whose reduction mod 17 is $\mu P_{1}+x R_{1}+y R_{2}$ for some $\lambda, \mu, u, v, x, y \in \mathbb{Z}$ with $(u, v) \neq(0,0)$ and $(x, y) \neq(0,0)$. Therefore the equation $\frac{2}{75}\left(7 u^{2}-12 u v+18 v^{2}\right)=\frac{1}{450}\left(139 x^{2}+76 x y+316 y^{2}\right)$ has a solution in rational numbers $u, v, x, y$ not all zero. However this quadratic form of rank 4 is not locally soluble over the 3 -adics. Therefore $\rho=28$.

Case $(\boldsymbol{N}, \boldsymbol{\varepsilon})=(\mathbf{1 0}, \mathbf{3})$. We already know that $\rho=28,29$ or 30 . Let $p=31$ or 37 . Since $p \equiv 1(\bmod 3)$ the reductions of the points in Tables 3 and 4 give us points $P_{1}, P_{2}, P_{3}, P_{4} \in$ $E_{p}\left(\mathbb{F}_{p}(T)\right)$. In addition when $p=31$ we have

$$
\begin{aligned}
& Q_{1}=\left(20 T^{4}+13 T^{3}+30 T^{2}+6 T, 5 T^{5}+4 T^{4}+29 T^{3}+4 T^{2}+5 T\right), \\
& \left.Q_{2}=\left(7 T^{6}+12 T^{5}+9 T^{4}+19 T^{2}+13 T+4\right) /(T+29)^{2}, \ldots\right),
\end{aligned}
$$

and when $p=37$ we have

$$
\begin{aligned}
& R_{1}=\left(36 T^{4}+11 T^{3}+4 T^{2}, 26 T^{5}+34 T^{4}+2 T^{3}+15 T^{2}\right), \\
& R_{2}=\left(6 T^{4}+5 T^{3}+T^{2}+26 T+32,29 T^{5}+35 T^{4}+2 T^{3}+15 T^{2}+19 T+10\right) .
\end{aligned}
$$

4 There is presumably a systematic reason for this, similar to that described in [5]. 
Using either Lemma 4.1 or Lemma 4.2 we find that $\Delta_{31}=2 \cdot 5$ and $\Delta_{37}=1$. Therefore $\rho \leq 29$.

As in the previous example, reducing mod 31 or 37 does not change the singular fibres. We compute

$$
\begin{aligned}
\operatorname{Reg}\left(P_{1}, P_{2}, P_{3}, P_{4}, u Q_{1}+v Q_{2}\right) & =\frac{5}{96}\left(25 u^{2}-4 u v+52 v^{2}\right), \\
\operatorname{Reg}\left(P_{1}, P_{2}, P_{3}, P_{4}, x R_{1}+y R_{2}\right) & =\frac{1}{8}\left(5 x^{2}+8 y^{2}\right) .
\end{aligned}
$$

If $\rho=29$ then the equation $\frac{5}{96}\left(25 u^{2}-4 u v+52 v^{2}\right)=\frac{1}{8}\left(5 x^{2}+8 y^{2}\right)$ has a solution in rational numbers $u, v, x, y$ not all zero. However this quadratic form is not locally soluble over the 3-adics. Therefore $\rho=28$.

Case $(\boldsymbol{N}, \boldsymbol{\varepsilon})=(\mathbf{1 1}, \mathbf{1})$. We already know that $\rho=28,29$ or 30 . Let $P_{1}$ and $P_{2}$ be the reductions mod $p$ of the points in Table 3. In addition, when $p=23$ we have

$$
\begin{aligned}
& Q_{1}=\left(16 T^{2}+5 T+5,21 T^{3}+15 T^{2}+3 T+18\right), \\
& \left.Q_{2}=\left(18 T^{6}+5 T^{5}+5 T^{4}+22 T^{3}+9 T^{2}\right) /(T+16)^{2}, \ldots\right),
\end{aligned}
$$

and when $p=53$ we have

$$
\begin{aligned}
& R_{1}=\left(28 T^{5}+T^{4}+23 T^{3}+40 T^{2}+15 T, \ldots\right), \\
& \left.R_{2}=\left(49 T^{6}+44 T^{5}+38 T^{4}\right) /\left(T^{2}+42 T+5\right)^{2}, \ldots\right) .
\end{aligned}
$$

Using either Lemma 4.1 or Lemma 4.2 we find that $\Delta_{23}=2 \cdot 7 \cdot 11 \cdot 13$ and $\Delta_{53}=11 \cdot 131$. Therefore $\rho \leq 29$.

Again, reducing mod 23 or 53 does not change the singular fibres. We compute

$$
\begin{aligned}
\operatorname{Reg}\left(P_{1}, P_{2}, u Q_{1}+v Q_{2}\right) & =\frac{11}{480}\left(57 u^{2}-46 u v+137 v^{2}\right), \\
\operatorname{Reg}\left(P_{1}, P_{2}, x R_{1}+y R_{2}\right) & =\frac{1}{240}\left(541 x^{2}-228 x y+1196 y^{2}\right) .
\end{aligned}
$$

If $\rho=29$ then the equation $\frac{11}{480}\left(57 u^{2}-46 u v+137 v^{2}\right)=\frac{1}{240}\left(541 x^{2}-228 x y+1196 y^{2}\right)$ has a solution in rational numbers $u, v, x, y$ not all zero. However this quadratic form is not locally soluble over the 11 -adics. Therefore $\rho=28$.

Open Access This article is distributed under the terms of the Creative Commons Attribution 4.0 International License (http://creativecommons.org/licenses/by/4.0/), which permits unrestricted use, distribution, and reproduction in any medium, provided you give appropriate credit to the original author(s) and the source, provide a link to the Creative Commons license, and indicate if changes were made.

\section{References}

1. Artin, M., Swinnerton-Dyer, H.P.F.: The Shafarevich-Tate conjecture for pencils of elliptic curves on K3 surfaces. Invent. Math. 20, 249-266 (1973)

2. Barth, W.P., Hulek, K., Peters, C.A.M., Van de Ven, A.: Compact Complex Surfaces. Springer, Berlin (2004)

3. Bosma, W., Cannon, J., Playoust, C.: The Magma algebra system I: The user language, J. Symb. Comb. 24, 235-265 (1997). http://magma.maths.usyd.edu.au/magma/

4. Cassels, J.W.S.: Lectures on Elliptic Curves, London Mathematical Society Student Texts, 24. Cambridge University Press, Cambridge (1991)

5. Charles, F.: On the Picard number of K3 surfaces over number fields. Algebra Numb. Theory 8(1), 1-17 (2014) 
6. Chen, I.: On Siegel's modular curve of level 5 and the class number one problem. J. Numb. Theory 74(2), 278-297 (1999)

7. Chen, Z.: Congruences of elliptic curves, PhD thesis, University of Cambridge (2016). http://zc231.user. srcf.net/Maths/PhDThesis.pdf

8. Chen, Z.: Families of elliptic curves with the same mod 8 representation. Math. Proc. Camb. Philos. Soc. 165(1), 137-162 (2018)

9. Cremona, J.E., Mazur, B.: Visualizing elements in the Shafarevich-Tate group. Exp. Math. 9(1), 13-28 (2000)

10. Elkies, N.D.: Three lectures on elliptic surfaces and curves of high rank, Oberwolfach Reports (2007). arXiv:0709.2908 [math.NT]

11. Fisher, T.A.: The Hessian of a genus one curved. Proc. Lond. Math. Soc. (3) 104(3), 613-648 (2012)

12. Fisher, T.A.: Invariant theory for the elliptic normal quintic, I. Twists of X(5). Math. Ann. 356(2), 589-616 (2013)

13. Fisher, T.A.: On families of 7 and 11-congruent elliptic curves. LMS J. Comput. Math. 17(1), 536-564 (2014)

14. Fisher, T.A.: On families of 9-congruent elliptic curves. Acta Arith. 171(4), 371-387 (2015)

15. Frey, G.: On elliptic curves with isomorphic torsion structures and corresponding curves of genus 2 . In: Coates, J., Yau, S.-T. (eds.) Elliptic Curves, Modular Forms \& Fermat's Last Theorem (Hong Kong, 1993), pp. 79-98. Int. Press, Cambridge (1995)

16. Halberstadt, E.: Sur la courbe modulaire $X_{\text {ndép }}(11)$. Exp. Math. 7(2), 163-174 (1998)

17. Halberstadt, E., Kraus, A.: Sur la courbe modulaire $X_{E}$ (7). Exp. Math. 12(1), 27-40 (2003)

18. Huybrechts, D.: Lectures on K3 Surfaces, Cambridge Studies in Advanced Mathematics, 158. Cambridge University Press, Cambridge (2016)

19. Kani, E., Schanz, W.: Modular diagonal quotient surfaces. Math. Z. 227(2), 337-366 (1998)

20. Kloosterman, R.: Elliptic K3 surfaces with geometric Mordell-Weil rank 15. Can. Math. Bull. 50(2), 215-226 (2007)

21. Kumar, A.: Hilbert modular surfaces for square discriminants and elliptic subfields of genus 2 function fields. Res. Math. Sci 2, 46 (2015). Art. 24

22. Miranda, R.: The basic theory of elliptic surfaces, Dottorato di Ricerca in Matematica. ETS Editrice, Pisa (1989)

23. Naskręcki, B.: Mordell-Weil ranks of families of elliptic curves associated to Pythagorean triples. Acta Arith. 160(2), 159-183 (2013)

24. Papadopoulos, I.: Courbes elliptiques ayant même 6-torsion qu'une courbe elliptique donnée. J. Numb. Theory 79(1), 103-114 (1999)

25. Poonen, B., Schaefer, E.F., Stoll, M.: Twists of $X(7)$ and primitive solutions to $x^{2}+y^{3}=z^{7}$. Duke Math. J. 137(1), 103-158 (2007)

26. Rubin, K., Silverberg, A.: Families of elliptic curves with constant mod $p$ representations. In: Coates, J., Yau, S.-T. (eds.) Elliptic Curves, Modular Forms \& Fermat's Last Theorem (Hong Kong, 1993), pp. 148-161. Int. Press, Cambridge (1995)

27. Rubin, K., Silverberg, A.: Mod 6 representations of elliptic curves, In: Doran, R.S., Dou,Z.-L., Gilbert, G.T. (eds.) Automorphic forms, automorphic representations, and arithmetic (Fort Worth, TX, 1996), Proc. Sympos. Pure Math., 66, Part 1, Amer. Math. Soc., Providence, RI, pp. 213-220 (1999)

28. Rubin, K., Silverberg, A.: Mod 2 representations of elliptic curves. Proc. Am. Math. Soc. 129(1), 53-57 (2001)

29. Shioda, T.: On the Mordell-Weil lattices. Comment. Math. Univ. St. Paul. 39(2), 211-240 (1990)

30. Silverberg, A.: Explicit families of elliptic curves with prescribed $\bmod N$ representations. In: Cornell, G., Silverman, J.H., Stevens, G. (eds.) Modular Forms and Fermat's Last Theorem (Boston, MA, 1995), pp. 447-461. Springer, New York (1997)

31. Swinnerton-Dyer, H.P.F.: Density of rational points on certain surfaces. Algebra Numb. Theory 7(4), 835-851 (2013)

32. Totaro, B.: Recent progress on the Tate conjecture. Bull. Am. Math. Soc. 54(4), 575-590 (2017)

33. van Luijk, R.: An elliptic K3 surface associated to Heron triangles. J. Numb. Theory 123(1), 92-119 (2007)

34. van Luijk, R.: K3 surfaces with Picard number one and infinitely many rational points. Algebra Numb. Theory 1(1), 1-15 (2007)

35. van Luijk, R.: Density of rational points on elliptic surfaces. Acta Arith. 156(2), 189-199 (2012)

Publisher's Note Springer Nature remains neutral with regard to jurisdictional claims in published maps and institutional affiliations. 